\title{
Évolution de la production laitière et du poids vif des vaches laitières au cours de leur carrière
}

\author{
JB Coulon ${ }^{1}$, L Pérochon 2 ${ }^{2}$ F Lescourret ${ }^{2}$ \\ 1 INRA-Theix, laboratoire Adaptation des herbivores aux milieux, \\ 63122 Saint-Genès-Champanelle; \\ 2 INRA-Theix, laboratoire d'écopathologie, 63122 Saint-Genès-Champanelle, France
}

(Reçu le 10 avril 1994 ; accepté le 27 juin 1994)

\begin{abstract}
Résumé - Cent soixante-seize vaches laitières ayant réalisé au moins 3 lactations consécutives dans des conditions d'alimentation non restrictives et sans troubles sanitaires au cours des 2 premières semaines de chacune de leurs lactations ont été utilisées pour décrire et analyser l'évolution de la production laitière initiale (moyenne des $4^{e}, 5^{e}$ et $6^{e}$ j de lactation) et maximale, et du poids vif au vêlage au cours des 3 premières lactations. En moyenne, la production initiale, la production maximale et le poids vif au vêlage sont passés de respectivement $15,7 \mathrm{~kg} / \mathrm{j}, 22,5 \mathrm{~kg} / \mathrm{j}$ et $536 \mathrm{~kg}$ en $1^{\text {re lactation à }}$ $21,7 \mathrm{~kg} / \mathrm{j}, 28,0 \mathrm{~kg} / \mathrm{j}$ et $576 \mathrm{~kg}$ en $2^{\mathrm{e}}$ lactation et $23,3 \mathrm{~kg} / \mathrm{j}, 29,8 \mathrm{~kg} / \mathrm{j}$ et $607 \mathrm{~kg}$ en $3^{e}$ lactation. Chez les 61 vaches ayant réalisé une $4^{e}$ lactation, le poids vif au vêlage a encore augmenté de $29 \mathrm{~kg}$ par rapport à la $3^{e}$ lactation, alors que la production initiale n'a pratiquement plus augmenté. Trois formes principales d'évolution de la production initiale et du poids vif ont été identifiées. Elles diffèrent essentiellement par la répartition de l'augmentation des performances entre les 2 premières lactation et entre les lactations 2 et 3 . Certains animaux n'augmentent leur production laitière ou leur poids vif qu'entre la $2^{e}$ et la $3^{e}$ lactation. L'augmentation de production laitière d'une lactation à l'autre a été d'autant plus importante que celle du poids vif l'était aussi ( $+2 \mathrm{~kg}$ de lait par $50 \mathrm{~kg}$ de gain de poids vif). Des équations de prévision des productions initiales et maximales des lactations 2 et 3 à partir des productions des lactations précédentes, de la race et des variations de poids vif sont proposées.
\end{abstract}

\section{vache laitière / lait / poids vif / numéro de lactation}

Summary - Pattern of milk production and live weight of dairy cows during their successive lactations. One hundred seventy-six dairy cows which had completed at least 3 lactations were used to describe and to analyse the pattern of initial milk production (mean of days 4, 5, and 6 of lactation), peak milk production and of live weight at calving during the first 3 lactations. These cows were free of health disorders during the first 2 weeks of each lactation and fed according to INRA recommendations. On average, initial production, peak production, and live weight at calving were 15.7 $\mathrm{kg} / \mathrm{d}, 22.5 \mathrm{~kg} / \mathrm{d}$ and $536 \mathrm{~kg}$ in 1st lactation, respectively; $21.7 \mathrm{~kg} / \mathrm{d}, 28.0 \mathrm{~kg} / \mathrm{d}$ and $576 \mathrm{~kg}$ in 2nd lactation; and $23.3 \mathrm{~kg} / \mathrm{d}, 29.8 \mathrm{~kg} / \mathrm{d}$ and $607 \mathrm{~kg}$ in 3 rd lactation. When a 4th lactation was completed, live weight was $29 \mathrm{~kg}$ higher than that of the 3rd lactation, whereas initial production did not increase signifi- 
cantly. Three main patterns were identified for both initial production and live weight. The increase of performance between the first 2 lactations and between the 2nd and 3rd lactation differed among patterns. For some cows, initial production or live weight increased only between the 2nd and 3rd lactation. The increase of milk production between 2 consecutive lactations was as important as that of live weight ( $+2 \mathrm{~kg}$ milk per $50 \mathrm{~kg}$ live weight). Equations were proposed to predict initial and peak milk productions for parities 2 and 3 on the basis of productions of preceding lactations, breed and live weight changes.

dairy cow / milk production / live weight / parity

\section{INTRODUCTION}

La gestion raisonnée des troupeaux laitiers nécessite la prise en compte de l'évolution des performances des animaux au cours de leurs lactations successives. L'étude de la forme et du déterminisme de ces évolutions est intéressante non seulement sur le plan cognitif, mais aussi pour la prévision précoce des performances individuelles à long terme. La production des vaches laitières lors de leur première lactation ne représente en moyenne que 75 à $85 \%$ de celle observée lors de leur lactation maximale (Hoden, 1978 ; Faverdin et al, 1987 ; Schultz et al, 1990 ; Ray et al, 1992) généralement atteinte en $3^{\mathrm{e}}$ ou $4^{\mathrm{e}}$ lactation. L'essentiel de cette augmentation a lieu entre la première et la deuxième lactation. Les écarts entre ces 2 lactations sont encore plus élevés si I'on considère la production maximale, en raison de la meilleure persistance de production des vaches primipares (Faverdin et al, 1987 ; Schultz et al, 1990), ou la production initiale (production moyenne des $4^{\mathrm{e}}, 5^{\mathrm{e}}$ et $6^{e} \mathrm{j}$ de lactation). Cette dernière est spécialement intéressante car elle est considérée comme un bon indicateur du potentiel des animaux (Decaen et al, 1970 ; Hoden et Journet, 1971 ; Hoden, 1978). L'augmentation de la production initiale entre la $1^{\text {re }}$ et la $2^{e}$ lactation varie selon les auteurs de 40 à $50 \%$ (Decaen et Poutous, 1965 ; Decaen et al, 1970 ; Hoden, 1978 ; Faverdin et al, 1987).

Au-delà de ces chiffres moyens, se pose le problème de la variabilité des types d'évolution des performances, et des sources de cette variabilité. Un certain nombre de travaux ont montré que la race des animaux (Oldenbroek, 1984 ; Mainland, 1985 ; Schultz et al, 1990), leur âge au premier vêlage (Schultz, 1960 ; Bettenay, 1985), ou leur alimentation au cours de la période d'élevage (Little et Kay, 1979) pouvaient influencer l'évolution des différents paramètres de production laitière d'une lactation à l'autre, en raison des différences dans le développement mammaire atteint au premier vêlage. Des différences ont aussi été observées selon la saison de vêlage (Ray et al, 1992). Ces évolutions, ainsi que celles du poids vif qui ont fait l'objet de beaucoup moins d'études (Chrichton et al, 1960a ; Schultz, 1960 ; Bettenay, 1985 ; Troccon, 1993), dépendent aussi des conditions d'alimentation des animaux au cours de leurs lactations successives (Oldenbroek, 1984 ; Coulon et al, 1990), ainsi que de leur état sanitaire. Cependant, dans la plupart de ces études, en particulier celles réalisées à partir d'un grand nombre de données issues du contrôle laitier, ces 2 derniers facteurs ont rarement été pris en compte.

L'objectif de ce travail a été dans un premier temps de décrire, chez des animaux les plus comparables possibles en matière de conduite alimentaire et de santé, l'évolution de la production laitière et du poids vif au cours des 3 ou 4 premières lactations et d'en analyser la variabilité en fonction des caractéristiques des animaux. Les critères de performances choisis, production initiale et poids vif au vêlage, sont ceux qui reflètent le mieux le potentiel des animaux et 
qui, compte tenu de leur connaissance très précoce au cours de la lactation, sont les plus intéressants sur le plan prévisionnel. Les résultats obtenus ont permis de proposer des équations de prédiction des productions laitières initiales et maximales au cours des lactations successives.

\section{MATÉRIEL ET MÉTHODES}

Les données utilisées dans cette étude ont été extraites de la base de données LASCAR qui regroupe les caractéristiques initiales et les performances réalisées au cours de la carrière (production, poids vif, reproduction, santé) de 1179 vaches conduites dans les domaines expérimentaux INRA de Theix, Marcenat et Orcival au cours des 20 dernières années (Lescourret et al, 1992 ; Coulon et al, 1993). Pour disposer d'animaux les plus comparables possibles d'une lactation à l'autre, on a sélectionné, parmi ces vaches, celles ayant réalisé 3 lactations successives $(n=176)$ dans les conditions suivantes: leurs vêlages devaient avoir eu lieu entre le $1^{\text {er }}$ octobre et le 31 mars, elles devaient avoir été indemnes de troubles sanitaires majeurs (avortement, non délivrance, panaris, mammites et fièvre vitulaire) au cours des 2 premières semaines de chacune des 3 lactations, et avoir reçu au cours des 2 premiers mois de chaque lactation des apports nutritifs énergétiques et azotés correspondant au moins aux recommandations alimentaires proposées par l'INRA (Hoden, 1978).

Les variables étudiées ont été la production initiale $(\mathrm{PI})$ et le poids vif mesuré au cours de la semaine suivant le vêlage (PV). Dans un premier temps, une typologie de l'évolution de chacun de ces 2 paramètres a été réalisée. Pour cela, une analyse en composantes principales (ACP) centrée réduite a été réalisée, prenant en compte la valeur de la variable étudiée en première lactation ( $\mathrm{Pl} 1$ ou $P V 1)$, et les écarts proportionnels pour les lactations suivantes ((PI2-PI1)/PI1, et (PI3-PI2)/PI2 par exemple pour la $\mathrm{PI}$ ), de manière à disposer de variables plus indépendantes entre elles que les valeurs brutes de la $\mathrm{PI}$ ou du poids vif. Ensuite, une classification ascendante hiérarchique à liens complets a été réalisée sur les coordonnées des individus sur l'ensemble des axes de l'ACP. Le nombre de classes retenues a été déterminé par l'examen de l'arbre obtenu. Les caractéristiques des vaches des différentes classes retenues ont été comparées. Il s'agissait en particulier de la race des animaux (Montbéliarde $(M O)$ ), Frisonne (FF), Holstein (HO), croisée HO*MO et croisée $\mathrm{HO}^{\star} \mathrm{FF}$ ), de l'index laitier de leur père (renseigné pour $61 \%$ des vaches uniquement), de leur âge au premier vêlage (inférieur à 26 mois, compris entre 26 et 33 mois, supérieur à 33 mois), des croissances observées entre la naissance et 6 mois et entre 6 mois et 1 an, et de l'état de développement corporel atteint au premier vêlage, estimé par le rapport des poids au vêlage entre la $1^{\text {re }}$ et la $3^{e}$ lactation. Ces 3 dernières variables permettent en effet de décrire de manière synthétique la phase d'élevage des animaux (Troccon et al, 1994). La signification des écarts entre les caractéristiques des différentes classes de $\mathrm{Pl}$ et de poids vif a été étudiée par analyse de variance pour les variables quantitatives et par le test du $\chi^{2}$ pour les variables qualitatives.

\section{RÉSULTATS}

\section{Caractéristiques de la population étudiée}

Les vaches retenues dans cette étude ont en majorité $(82 \%)$ vêlé pour la première fois à un âge supérieur à 26 mois. Aucun premier vêlage n'a eu lieu avant 23 mois. Elles étaient de race $\mathrm{MO}(22 \%), \mathrm{FF}(25 \%)$ et $\mathrm{HO}$ (25\%), ou croisées $\left(17 \%\right.$ de $\mathrm{HO}^{*} \mathrm{MO}$ et $11 \%$ de $\left.\mathrm{HO}^{\star} \mathrm{FF}\right)$. Leur production initiale a été en moyenne de $15,7,21,7$ et $23,3 \mathrm{~kg} / \mathrm{j}$ au cours des 3 lactations étudiées, leur production maximale de $22,5,28,0$ et $29,8 \mathrm{~kg} / \mathrm{j}$, et leur poids vifs au vêlage de 536,576 et $607 \mathrm{~kg}$. Ces valeurs ont présenté une variabilité élevée, de l'ordre de 20 à $25 \%$ pour les productions laitières et de $10 \%$ pour les poids vifs au vêlage. En moyenne, la $\mathrm{Pl}$ a augmenté de $5,9 \mathrm{~kg}( \pm 4,1)$ entre la $1^{\text {re }}$ et la $2^{\mathrm{e}}$ lactation et de $1,6 \mathrm{~kg}( \pm 4,4)$ entre la $2^{e}$ et la $3^{e}$ lactation. Les augmentations du poids vif au vêlage correspondantes ont été de 40 $( \pm 43)$ et $31( \pm 34 \mathrm{~kg})$. En moyenne, chez les vaches ayant réalisé une $4^{\mathrm{e}}$ lactation d'au moins $200 \mathrm{j}$ dans les mêmes conditions que les 3 précédentes $(n=61)$, seul le poids vif 
au vêlage a sensiblement augmenté entre la $3^{e}$ et la $4^{e}$ lactation $(+29 \mathrm{~kg})$. Le tableau I résume, pour cette population, l'évolution des principales performances, concernant le début de la lactation et la lactation totale, au cours des 4 premières lactations.

\section{Évolution de la production initiale (fig 1 et tableau II)}

Trois formes d'évolution de la PI ont été retenues à partir de la classification réalisée (fig 1). Une $4^{\mathrm{e}}$ classe, ne rassemblant que 4 vaches dont les PI étaient très faibles en première lactation, n'a pas été prise en compte dans l'analyse ultérieure. La classe 1 rassemble la majorité des vaches $(n=94)$. Elle est caractérisée par une $\mathrm{Pl}$ élevée en $1^{\text {re }}$ lactation (18,4 kg/j en moyenne), une augmentation moyenne entre la $1^{\text {re }}$ et la $2^{\mathrm{e}}$ lactation $(5,4 \mathrm{~kg})$ et faible ensuite $(1,1 \mathrm{~kg})$. Les classes $2(n=20)$ et $3(n=58)$ sont caractérisées par une faible $\mathrm{PI}$ en première lactation (respectivement 13,8 et $12,8 \mathrm{~kg} / \mathrm{j}$ en moyenne). Dans la classe 2, la P | n'augmente qu'entre les lactations 2 et $3(+7,2 \mathrm{~kg})$, à l'inverse de la classe 3 où elle n'augmente qu'entre les 2 premières $(+8,0 \mathrm{~kg})$. Les différences de production laitière à l'échelle de la lactation (production en 305 j) reflètent tout à fait celles de la production initiale. Ainsi, la production en $305 \mathrm{j}$ a en moyenne augmenté de $820 \mathrm{~kg}$ entre la $1^{\text {re }}$ et la $2^{\mathrm{e}}$ lactation dans la classe 3 , alors qu'elle n'a pas été modifiée dans la classe 2. Dans ces 2 classes, la production initiale en $3^{2}$ lactation représente en moyenne respectivement 158 et $168 \%$ de celle de la première lactation, contre $137 \%$ dans la classe 1 .

Ces 3 classes se différencient d'abord par le type génétique des animaux. Dans la classe 1 les animaux de race $\mathrm{HO}$ et $\mathrm{FF}^{\star} \mathrm{HO}$ constituent près de la moitié de l'effectif alors qu'ils ne représentent que $21 \%$ de la classe 3 et $30 \%$ de la classe $2(P<0,01)$. Cet effet n'est pas dû au domaine expérimental d'origine des animaux (auquel le type génétique est très lié), puisqu'il se maintient lorsque l'on réalise l'analyse intra-domaine (par exemple à Marcenat, respectivement 24 et $76 \%$ d'animaux $\mathrm{MO}$ et $\mathrm{FF}$ dans la classe 1 contre 61 et $39 \%$ dans la classe $3, P<0,01$ ). Les animaux de la classe 3 sont par ailleurs

Tableau I. Évolution des performances des vaches laitières au cours de leurs 4 premières lactations (61 vaches conduites au cours de leurs 4 premières lactations dans des conditions d'alimentation non restrictives, sans troubles sanitaires au cours des 2 premières semaines de lactation, et d'une durée de lactation toujours supérieure ou égale à $200 \mathrm{j}$ ).

\begin{tabular}{|c|c|c|c|c|}
\hline & \multicolumn{4}{|c|}{ Numéro de lactation } \\
\hline & 1 & 2 & 3 & 4 \\
\hline & & & & - \\
\hline Production initiale $(\mathrm{kg} / \mathrm{j})$ & 15,5 & 20,6 & 22,4 & 22,7 \\
\hline Production maximale $(\mathrm{kg} / \mathrm{j})$ & 21,9 & 27,1 & 29,0 & 30,3 \\
\hline Production $305 \mathrm{j}(\mathrm{kg})$ a & 4613 & 5287 & 5542 & 5553 \\
\hline Durée de lactation (j) & 298 & 305 & 300 & 297 \\
\hline Taux butyreux $(\mathrm{g} / \mathrm{kg})^{b}$ & 39,0 & 39,8 & 38,7 & 39,0 \\
\hline Taux protéique $(\mathrm{g} / \mathrm{kg})^{\mathrm{b}}$ & 31,7 & 31,8 & 31,4 & 31,7 \\
\hline Poids vif au vêlage (kg) & 534 & 564 & 598 & 627 \\
\hline
\end{tabular}

\footnotetext{
a Production laitière cumulée sur les 305 premiers jours de lactation si la durée de lactation est supérieure à $305 \mathrm{j}$, et sur la lactation totale si la durée de lactation est inférieure à $305 \mathrm{j} ;{ }^{b}$ moyenne pondérée sur la lactation totale.
} 

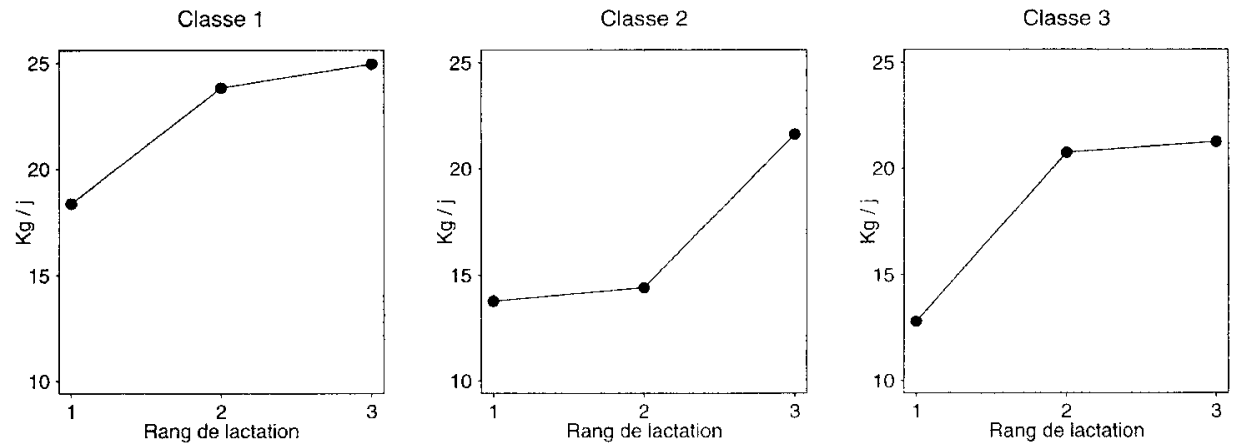

Fig 1. Évolution moyenne de la production initiale au cours des 3 premières lactations dans les 3 classes réalisées sur la $\mathrm{PI}$.

plus légers au premier vêlage que ceux des classes 1 et 2 (de respectivement 32 et $20 \mathrm{~kg}, P<0,01$ ), en relation avec une proportion un peu plus importante de vêlages précoces $(26 \%$ de vêlages avant 26 mois contre respectivement 10 et $14 \%, P<0,1$ ) ; ils présentent un gain de poids vif entre les 2 premiers vêlages un peu supérieur $(P<$ $0,1)$ à celui des 2 autres classes. Les vaches de la classe 2 ont présenté une durée de tarissement en première lactation plus courte que celles des autres classes $(P<0,05)$. En revanche, aucune différence significative n'a été observée entre les 3 classes entre les croissances durant la première année ou le développement atteint au premier vêlage (tableau II). Chez les vaches FF pour lesquelles l'index laitier du père était renseigné ( $n=68$ ), cet index n'a pas été différent d'une classe à l'autre. Dans ces 3 classes, chez les vaches ayant commencé une quatrième lactation ( $n=67$ ), l'évolution de la PI entre la $3^{e}$ et la $4^{e}$ lactation a été faible (respectivement $+0,6,+0,2$ et $+1,4 \mathrm{~kg}$ ).

\section{Évolution du poids vif (fig 2 et tableau III)}

Trois formes d'évolution du poids vif au vêlage ont été retenues à partir de la clas- sification réalisée (fig 2). Les classes 1 ( $n=$ $74)$ et $2(n=84)$ regroupent des vaches assez légères au premier vêlage (respectivement 535 et $521 \mathrm{~kg}$ ), augmentant fortement de poids entre la $1^{\text {re }}$ et la $2^{\mathrm{e}}$ lactation (respectivement +57 et $+39 \mathrm{~kg}$ ). Elles se différencient par l'augmentation de poids vif entre la deuxième et la troisième lactation, faible dans la classe $1(+6 \mathrm{~kg})$ et forte dans la classe $2(+46 \mathrm{~kg})$. La classe $3(n=18)$ est caractérisée au contraire par des animaux très lourds au 1 er vêlage $(616 \mathrm{~kg})$, plus légers de $27 \mathrm{~kg}$ au $2^{\mathrm{e}}$ vêlage qu'au $1^{\mathrm{er}}$ et gagnant ensuite $56 \mathrm{~kg}$ au troisième vêlage. Dans cette classe, le poids vif au troisième vêlage ne représente que 105\%

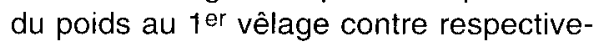
ment 111 et $116 \%$ dans les classes 1 et 2 $(P<0,01)$.

Ces 3 classes ne se différencient pas par la race des animaux (tableau III). La PI en $1^{\text {re }}$ lactation est supérieure dans la classe 3 (19,1 contre 15,3 kg/j). Cet écart se maintient en $3^{e}$ lactation, de sorte que le rapport PI3/PI1 a été peu différent d'une classe à l'autre (respectivement $1,45,1,49$ et 1,38 pour les classes 1, 2 et 3 ). La croissance des animaux a été légèrement supérieure chez les animaux de la classe 3 au cours de leurs 6 premiers mois (d'environ $60 \mathrm{~g} / \mathrm{j}$, $P<0,05)$, mais semblable entre 6 mois et 1 
an. C'est donc entre 1 an et le 1 er vêlage que les croissances ont été les plus différentes entre les vaches de la classe 3 et celles des classes 1 et 2 , en raison d'un âge au $1^{\text {er }}$ vêlage beaucoup plus tardif dans cette classe où tous les animaux ont vêlé pour la première fois après 33 mois. Chez les vaches ayant commencé une $4^{e}$ lactation, le poids vif a augmenté entre la $3^{e}$ et la $4^{4}$ lactation, de manière voisine dans les 3 classes (respectivement $+27,+21$ et $+35 \mathrm{~kg})$.

Tableau II. Caractéristiques moyennes des 3 classes d'évolution de la production initiale (PI).

\begin{tabular}{|c|c|c|c|c|c|}
\hline & 1 & $\begin{array}{c}\text { Classe } \\
2 \\
2\end{array}$ & 3 & ETR a & \\
\hline $\mathrm{N}$ & 94 & 20 & 58 & & \\
\hline Pl lactation 1 & 18,4 & 13,8 & 12,8 & 2,5 & ** \\
\hline $\begin{array}{l}\text { Variation de PI }(\mathrm{kg}) \\
\text { Lactation } 2 \text { - lactation } 1 \\
\text { Lactation } 3 \text { - lactation } 2\end{array}$ & $\begin{array}{l}5,4 \\
1,1\end{array}$ & $\begin{array}{l}0,6 \\
7,2\end{array}$ & $\begin{array}{l}8,0 \\
0,5\end{array}$ & $\begin{array}{l}3,3 \\
3,9\end{array}$ & $\begin{array}{l}* \star \\
\star \star\end{array}$ \\
\hline $\begin{array}{l}\text { Production laitière en } 305 \text { jours } \\
\text { Lactation } 1 \\
\text { Lactation } 2 \text { - lactation } 1 \\
\text { Lactation } 3 \text { - lactation } 2\end{array}$ & $\begin{array}{c}5352 \\
423 \\
238\end{array}$ & $\begin{array}{l}4507 \\
-4 \\
900\end{array}$ & $\begin{array}{l}4172 \\
824 \\
196\end{array}$ & $\begin{array}{l}949 \\
683 \\
770\end{array}$ & $\begin{array}{l}\star \star \\
\star * \\
* *\end{array}$ \\
\hline Poids vif (PV) au premier vêlage $(\mathrm{kg})$ & 549 & 537 & 517 & 55 & ** \\
\hline $\begin{array}{l}\text { Variation de poids vif }(\mathrm{kg}) \\
\text { Lactation } 2 \text { - lactation } 1 \\
\text { Lactation } 3 \text { - lactation } 2\end{array}$ & $\begin{array}{l}33 \\
30\end{array}$ & $\begin{array}{l}36 \\
46\end{array}$ & $\begin{array}{l}49 \\
24\end{array}$ & $\begin{array}{l}41 \\
34\end{array}$ & $\begin{array}{l}\star \star \star \\
\star \star \star\end{array}$ \\
\hline PV lactation 1/PV lactation 3 & 0,90 & 0,88 & 0,88 & 0,07 & NS \\
\hline Croissance entre la naissance et 6 mois $(g / j)$ & 712 & 733 & 710 & 92 & NS \\
\hline Croissance entre 6 mois et 1 an $(g / j)$ & 661 & 653 & 660 & 123 & NS \\
\hline $\begin{array}{l}\text { Race b } \\
\qquad \begin{array}{l}\mathrm{MO} \\
\mathrm{FF}+\mathrm{HO}^{\star} \mathrm{MO} \\
\mathrm{HO}+\mathrm{HO}^{\star} \mathrm{FF}\end{array}\end{array}$ & $\begin{array}{c}7 \\
42 \\
45\end{array}$ & $\begin{array}{l}6 \\
8 \\
6\end{array}$ & $\begin{array}{l}23 \\
23 \\
12\end{array}$ & & ** \\
\hline $\begin{array}{l}\text { Âge au premier vêlage } \\
\qquad=800 \mathrm{j} \\
800-1000 \mathrm{j} \\
>1000 \mathrm{j}\end{array}$ & $\begin{array}{l}13 \\
18 \\
63\end{array}$ & $\begin{array}{c}2 \\
7 \\
11\end{array}$ & $\begin{array}{l}15 \\
13 \\
30\end{array}$ & & $\star \star * *$ \\
\hline Durée de tarissement en lactation 1 (j) & 71 & 60 & 75 & 20 & * \\
\hline Durée de tarissement en lactation 2 (j) & 71 & 78 & 77 & 22 & NS \\
\hline
\end{tabular}



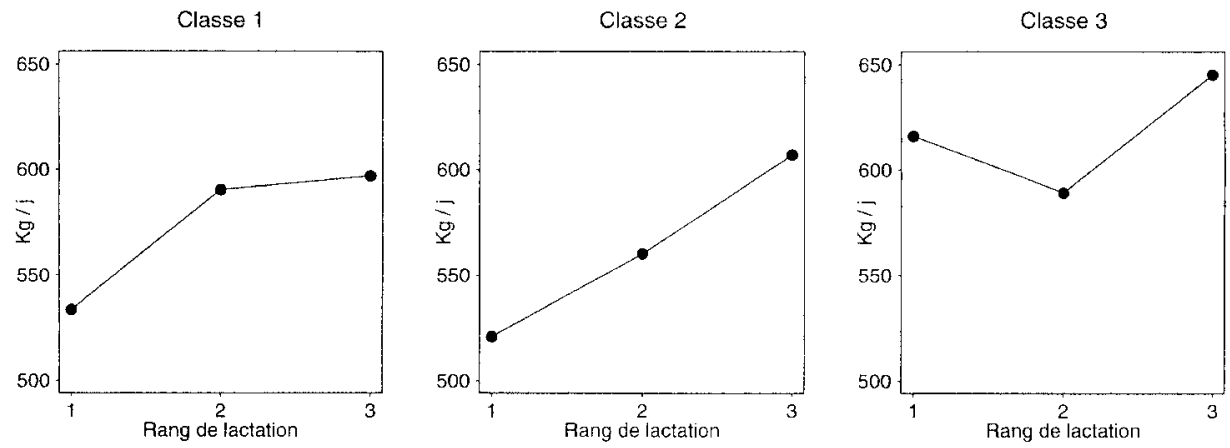

Fig 2. Évolution moyenne du poids vif au vêlage au cours des 3 premières lactations dans les 3 classes réalisées sur le poids vif.

\section{Prévision des productions initiale et maximale des lactations de rang 2 et 3}

L'analyse des 2 classifications montre que les évolutions de la PI et du poids vif au vêlage au cours de la carrière des animaux ne sont pas indépendantes, et révèle l'effet de certains facteurs de variation liés à l'animal (race) ou à sa conduite (âge au premier vêlage, très lié au poids au premier vêlage). Ces divers éléments peuvent être pris en compte pour prévoir les performances de production laitière des vaches au cours de leurs lactations successives.

Compte tenu de l'effet dépréciateur sensible d'une diminution de la durée de tarissement sur la production de la lactation suivante (Sorensen et Enevoldsen, 1991) et du nombre insuffisant de vaches ayant présenté des durées très courtes pour estimer cet effet avec précision, la prévision des productions initiales et maximales des lactations 2 et 3 a été réalisée en éliminant les animaux ayant des durées de tarissement inférieures à $40 \mathrm{j}$ (soit respectivement 4 et 1 vaches en lactation 1 et 2). Par ailleurs, les 4 vaches ayant présenté des PI anormalement faibles en 1 re lactation ( $c f$ résultats) n'ont pas été prises en compte pour la prévision de la $\mathrm{Pl}$ en $2^{\mathrm{e}}$ lactation.
La production initiale en $2^{e}$ lactation (PI2) ou en $3^{e}$ lactation (PI3) peut être estimée à partir de la production initiale des lactations précédentes par les relations suivantes:

$$
\begin{gathered}
\mathrm{Pl} 2=8,1+0,86 \mathrm{Pl} 1 \pm 3,8 \quad \mathrm{R}^{2}=0,41 \quad[1] \\
\mathrm{Pl} 3=7,4+0,35 \mathrm{Pl} 1+0,48 \mathrm{Pl} 2 \pm 3,6 \quad \mathrm{R}^{2}=0,48[2]
\end{gathered}
$$

La prise en compte de la classe d'évolution de $\mathrm{PI}$ et/ou de poids vif permet d'améliorer sensiblement la précision de ces équations (diminution de l'écart type résiduel de respectivement 0,8 et $0,4 \mathrm{~kg} / \mathrm{j}$ ). Cependant, en pratique, la prévision précoce de l'appartenance à une classe d'évolution de PI ou de poids vif est difficile à mettre en œuvre. Il est donc préférable de prendre directement en compte dans les équations de prévision les variables explicatives de ces profils d'évolution au fur et à mesure qu'elles deviennent disponibles.

Dans un premier temps (équations 3 et 4) on a introduit la race dans les équations de prédiction, et dans un second temps la variation de poids vif au cours de la lactation précédente (dPV12 et dPV23), et le poids vif au vêlage précédent ( $P V 1$ et $P V 2$ ) (équations 5 et 6 ). La prise en compte de l'âge au premier vêlage n'améliore alors pas significativement la prévision en raison de sa forte liaison avec le poids au premier vêlage. Les équations sont les suivantes: 
Tableau III. Caractéristiques moyennes des 3 classes d'évolution du poids vif au vêlage (PV).

\begin{tabular}{|c|c|c|c|c|c|}
\hline & & Classe & & ETR ${ }^{\mathrm{a}}$ & \\
\hline & 1 & 2 & 3 & & \\
\hline $\mathrm{N}$ & 74 & 84 & 18 & & \\
\hline PI b lactation $1(\mathrm{~kg} / \mathrm{j})$ & 15,5 & 15,2 & 19,1 & 3,7 & ** \\
\hline Variation de PI $(\mathrm{kg})$ & & & & & \\
\hline Lactation 2 - lactation 1 & 7,0 & 5,2 & 4,1 & 4,0 & $\star \star \star$ \\
\hline Lactation 3 - lactation 2 & 0,2 & 2,5 & 3,3 & 4,2 & $\star \star$ \\
\hline Poids vif au premier vêlage $(\mathrm{kg})$ & 534 & 521 & 616 & 49 & 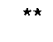 \\
\hline Variation de poids vif $(\mathrm{kg})$ & & & & & \\
\hline Lactation 2 - lactation 1 & 57 & 39 & -27 & 36 & $\star \star$ \\
\hline Lactation 3 - lactation 2 & 6 & 47 & 56 & 27 & $\star \star$ \\
\hline PV lactation 1/PV lactation 3 & 0,90 & 0,86 & 0,96 & 0,06 & ** \\
\hline Croissance entre la naissance et 6 mois $(\mathrm{g} / \mathrm{j})$ & 716 & 699 & 770 & 90 & * \\
\hline Croissance entre 6 mois et 1 an $(\mathrm{g} / \mathrm{j})$ & 680 & 649 & 623 & 121 & NS \\
\hline Race $^{c}$ & & & & & NS \\
\hline MO & 15 & 22 & 1 & & \\
\hline $\mathrm{FF}+\mathrm{HO}^{\star} \mathrm{MO}$ & 30 & 35 & 9 & & \\
\hline $\mathrm{HO}+\mathrm{HO}^{*} \mathrm{FF}$ & 29 & 27 & 8 & & \\
\hline Âge au premier vêlage & & & & & $\star \star$ \\
\hline$<=800 \mathrm{j}$ & 15 & 16 & 0 & & \\
\hline $800-1000 \mathrm{j}$ & 14 & 25 & 0 & & \\
\hline$>1000 \mathrm{j}$ & 45 & 43 & 18 & & \\
\hline Durée de tarissement en lactation 1 (j) & 75 & 71 & 59 & 19 & * \\
\hline Durée de tarissement en lactation 2 (j) & 74 & 76 & 68 & 22 & NS \\
\hline
\end{tabular}

a Écart type résiduel ; ${ }^{\mathrm{P}} \mathrm{PI}$ : production initiale ; ${ }^{\mathrm{C}} \mathrm{MO}$ : Montbéliarde ; FF : Française Frisonne ; HO : Holstein ; ${ }^{* *}: P<0,01 ;{ }^{*}: P<0,05 ;$ NS : non significatif.

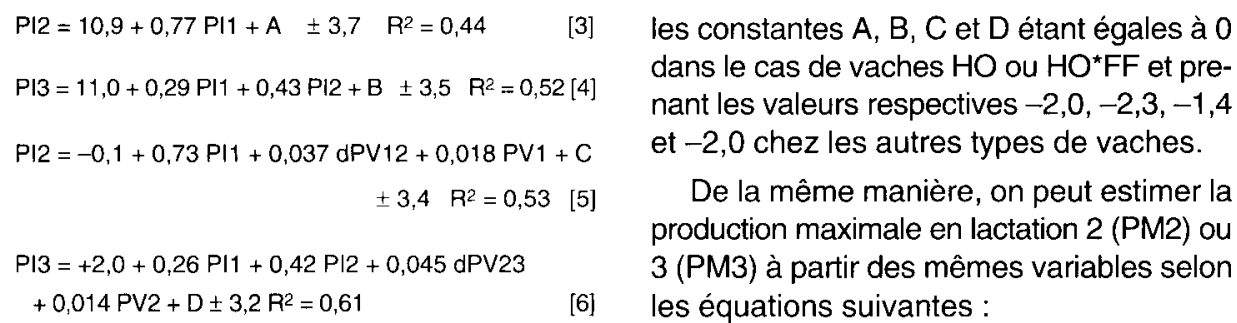


$\mathrm{PM} 2=8,4+0,88 \mathrm{PM} 1 \pm 3,7 \mathrm{R}^{2}=0,55$

$\mathrm{PM} 3=4,4+0,47 \mathrm{PM} 1+0,54 \mathrm{PM} 1+0,54 \mathrm{PM} 2$

$$
\pm 3,5 \quad R^{2}=0,66
$$

$P M 2=12,7+0,76 P M 1+E \pm 3,5 \quad R^{2}=0,59$

$P M 3=7,1+0,45 \mathrm{PM}^{2}+0,49 \mathrm{PM} 2+\mathrm{F}$

$$
\pm 3,4 \quad R^{2}=0,67
$$

$P M 2=2,7+0,74 P M 1+0,035 d P V 12+0,016 P V 1+G$

$$
\pm 3,3 \quad R^{2}=0,66 \quad[11]
$$

$\mathrm{PM} 3=5,5+0,39 \mathrm{PM} 1+0,55 \mathrm{PM} 2+0,040 \mathrm{dPV} 23+\mathrm{H}$

$$
\pm 3,2 \quad R^{2}=0,71
$$

les constantes $E, F, G$ et $H$ étant égales à 0 dans le cas de vaches $\mathrm{HO}$ ou HO*FF et prenant les valeurs respectives $-2,6,-1,4,-2,0$ et $-1,2$ chez les autres types de vaches.

La figure 3 illustre la relation entre PM2 et $\mathrm{PM} 1$, selon la race des animaux.

\section{DISCUSSION}

En moyenne, les augmentations de la production initiale et de la production maximale obtenues dans cette étude (respectivement $+5,9$ et $+5,6 \mathrm{~kg}$ entre les 2 premières lactations, soit +38 et $+25 \%$ et $+1,6$ et $+2,0 \mathrm{~kg}$ entre la $2^{\mathrm{e}}$ et la $3^{\mathrm{e}}$ lactation, soit +7 et $+7 \%$ ) sont voisines de celles observées par la plupart des auteurs (Decaen et al, 1970 ; Hoden, 1978 ; Mainland, 1985 ; Schultz et al, 1990). Ces augmentations ont cependant présenté une variabilité très importante. Les différentes formes d'évolution de la PI que nous avons obtenues montrent que pour certains animaux il est nécessaire d'attendre la $3^{\mathrm{e}}$ lactation pour avoir une bonne expression du potentiel de production adulte. Ces observations confirment le statut particulier de la $2^{e}$ lactation, souvent mis en évidence aussi bien du point de vue de l'appétit des animaux (Journet et al, 1965) que de leurs performances de production ou de leur état sanitaire (Faye et al, 1986 ; Coulon et al, 1990). Ceci est encore plus vrai pour le poids vif qui, dans près de la moitié des cas, augmente plus entre la $2^{e}$ et la $3^{e}$ lactation qu'entre les 2 premières, comme cela a été observé par Coulon et al (1990), mais uniquement chez des animaux sous-alimentés au cours de leurs 4 premiers mois de lactation. Cette étude a aussi mis en évidence que, même chez des vaches ayant en majorité réalisé leur premier vêlage à 3 ans, et conduites dans des conditions satisfaisantes d'alimentation, le poids vif au vêlage continue d'augmenter de manière sensible en $4^{\mathrm{e}}$ lactation, comme cela est couramment observé chez des vaches ayant vêlé pour la première fois à 2 ans ou moins (Crichton et al, 1960b ; Schultz, 1960 ; Troccon, 1993). L'évolution du poids vif des vaches ayant vêlé pour la première fois après 2 ans ( 30 à 33 mois) a fait l'objet de moins de travaux, et les résultats de ces travaux sont contradictoires (Schultz, 1960 ; Bettenay, 1985). Il est vraisemblable que ce gain de poids ne soit constitué qu'en faible partie par des dépôts adipeux, et témoigne d'une poursuite de la croissance jusqu'à un âge assez avancé (Crichton et al, 1960a). En effet, bien que nous ne disposions pas de l'état corporel des animaux dans cette étude, nous savons que la plupart d'entre eux n'ont jamais été fortement sur-alimentés au cours de leurs lactations successives.

La variabilité de l'évolution de la Pl dépend d'abord du niveau de cette production : la production augmente d'autant moins qu'elle était plus élevée au cours de la lactation précédente. Par ailleurs, les évolutions de la production laitière et du poids vif n'ont pas été indépendantes. En particulier, l'augmentation de production initiale ou maximale entre les lactations 1 et 2 ou 2 et 3 a été d'autant plus importante que la prise de poids a été plus élevée : un gain de poids de $50 \mathrm{~kg}$ entre 2 lactations se traduit par une augmentation de la production initiale ou maximale d'environ $2 \mathrm{~kg}$ $(P<0,01)$. Ceci doit résulter i) d'un développement du tissu mammaire, qui semble pouvoir se poursuivre au-delà du premier 


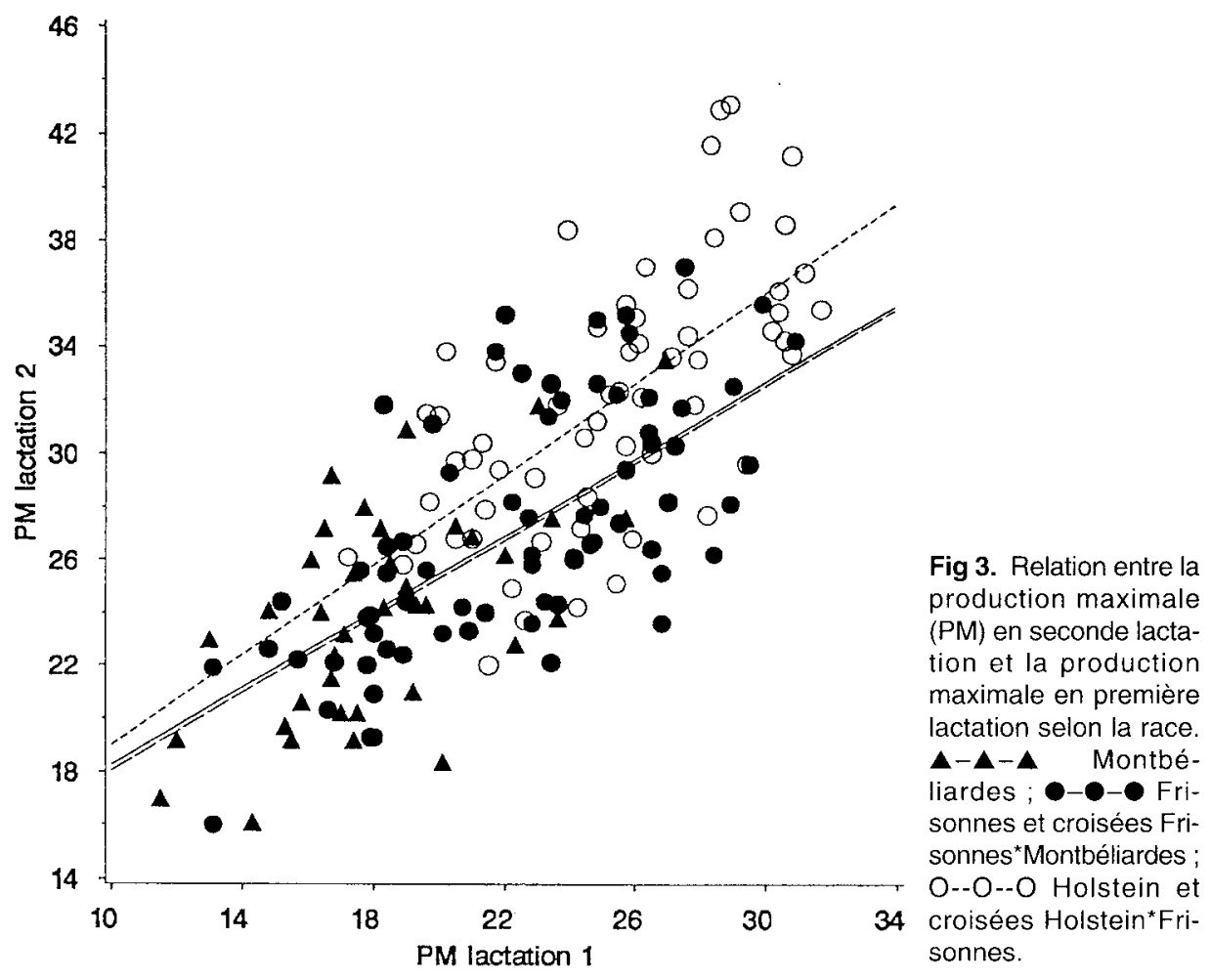

vêlage (Knight et Peaker, 1982 ; Knight et Wilde, 1993), et dont le gain de poids peut être un indicateur indirect ; ii) d'un meilleur état général des animaux, permettant en particulier un développement de la capacité d'ingestion et donc une meilleure faculté de faire face aux besoins de production et conduisant à une extériorisation du potentiel supérieure.

Les équations de prévision proposées montrent que les productions laitières initiale et maximale des lactations 2 et 3 ne sont pas directement proportionnelles à celle de la première lactation. Les coefficients de proportionnalité calculés sur la moyenne de la population tendent à surestimer fortement la production des vaches d'un niveau initial élevé (par exemple, d'environ $4 \mathrm{~kg}$ pour une production maximale en première lactation de $35 \mathrm{~kg} / \mathrm{j}$ ) et à sous- estimer celles d'un niveau initial faible (d'environ $3 \mathrm{~kg}$ pour une production maximale en première lactation de $20 \mathrm{~kg}$ ). Si la connaissance des variations de poids permet d'améliorer sensiblement la prévision de la production, en pratique elles sont rarement disponibles, et seule la correction pour l'effet race pourra être utilisé (équations [3] et [4] pour la PI, et [9] et [10] pour la production maximale). Ces équations permettent d'avoir une estimation précoce, satisfaisante en moyenne à l'échelle d'un lot de vaches, de la production des lactations ultérieures. Bien que couvrant déjà une large gamme de production (15 à $30 \mathrm{~kg}$ de production maximale en première lactation, fig 3 ), elles sont à confirmer dans des conditions de conduites plus intensives des animaux (vêlages précoces, potentiels de production élevés...). À terme, elles 
participeront à la construction d'un modèle de prévision des performances des vaches laitières à l'échelle de la carrière (Coulon et al, 1993).

\section{RÉFÉRENCES}

Bettenay RA (1985) Effect of growth rate and mating age of dairy heifers on subsequent production over 4 years. Aust $J$ Exp Agric 25, 263-269

Coulon JB, Landais E, Garel JP (1990) Alimentation, pathologie et productivité de la vache laitière : interrelations à l'échelle de la carrière. Ann Rech Vét21, $33-47$

Coulon JB, Lescourret F, Faye B, Landais E, Troccon JL, Pérochon $L$ (1993) Description de la base de données LASCAR, un outil pour l'étude des carrières des vaches laitières. INRA Prod Anim 6, 151-160

Crichton JA, Aitken JN, Boyne AW (1960a) The effect of plane of nutrition during rearing on growth, production, reproduction and health of dairy cattle. II. Growth to maturity. Anim Prod 2, 45-57

Crichton JA, Aitken JN, Boyne AW (1960b) The effect of plane of nutrition during rearing on growth production, reproduction and health of dairy cattle. III. Milk production during the first 3 lactations. Anim Prod 2, 159-168

Decaen C, Poutous M (1965) Phase ascendante de la courbe de lactation chez la vache laitière. Ann Zootech 14, 135-143

Decaen C, Calomiti S, Poutous M (1970) Évolution de la production laitière de la vache au cours des 2 premiers mois de la lactation. II. Analyse de la variation de la quantité de lait. Ann Zootech 19, 205221

Faverdin P, Hoden A, Coulon JB (1987) Recommandations alimentaires pour les vaches laitières. Bull Tech CRZV Theix INRA 70, 133-152

Faye B, Fayet JC, Brochart M, Barnouin J, Paccard P (1986) Enquête éco-pathologique continue. V. Mise en évidence des associations pathologiques en élevage bovin laitier : données individuelles. Ann Rech Vét 17, 265-286

Hoden A (1978) Rationnement au début de la lactation. In : La vache laitière, INRA Publications, 71-86
Hoden A, Journet M (1971) Le rationnement des vaches laitières au début de la lactation. Bull Tech CRZV Theix INRA 5, 5-28

Journet M, Poutous M, Calomiti S (1965) Appétit de la vache laitière. I. Variations ind ividuelles des quantités d'aliments ingérées. Ann Zootech 14, 5-37

Knight CH, Peaker M (1982) Development of the mammary gland. J Reprod Fert 65, 521-536

Knight CH, Wilde CJ (1993) Mammary cell changes during pregnancy and lactation. Livest Prod Sci 35, 3-19

Lescourret F, Pérochon L, Coulon JB, Faye B, Landais $E$ (1992) Modelling an information system using the MERISE method for agricultural research: the example of a database for a study on performances in dairy cows. Agric Syst 38, 149-173

Little W, Kay RM (1979) The effects of rapid rearing and early calving on subsequent performance of dairy heifers. Anim Prod 29, 131-142

Mainland DD (1985) A note on lactation curves of dairy cows in Scotland. Anim Prod 41, 413-416

Oldenbroek JK (1984) Holstein friesians, Dutch friesians and Dutch red and whites on 2 complete diets with a different amount of roughage: differences in performance between first and second lactation. Livest Prod Sci 11, 417-428

Ray DE, Halbach TJ, Armstrong DV (1992) Season and lactation number effects on milk production and reproduction of dairy cattle in Arizona. J Dairy Sci $75,2976-2983$

Schultz LH (1960) Relationship of rearing rate of dairy heiters to mature performance. J Dairy Sci 52, 1321-1329

Schultz MM, Hansen LB, Steuernagel GR, Kuck AL (1990) Variation of milk, fat, protein, and somatic cells for dairy cattle. J Dairy Sci 73, 484-493

Sorensen JT, Enevoldsen C (1991) Effect of dry period length on milk production in subsequent lactation. J Dairy Sci 74, 1277-1283

Troccon JL (1993) Effets de l'alimentation hivernale des génisses sur les performances et la longévité des vaches. INRA Prod Anim 6. 345-356

Troccon JL, Rulquin H, Journet M (1979) Capacités d'ingestion comparées des vaches primipares et multipares. Bull Tech CRZV Theix INRA 36, 43-48

Troccon JL, Coulon JB, Lescourret F (1994) Carrière des vaches laitières : caractérisation de la phase d'élevage et relation avec les performances en première lactation. INRA Prod Anim 7, 359-368 